\title{
Introduction : Entre fractures territoriales, représentations et identités culturelles: Comment appréhender le Nord britannique?
}

Spatial Divides, Cultural Representations and Identities: Exploring the Meaning

of the North in Great Britain

Mark Bailoni et Corinne Nativel

\section{(2) OpenEdition}

Journals

Édition électronique

URL : http://journals.openedition.org/rfcb/5631

DOI : $10.4000 / \mathrm{rfcb} .5631$

ISSN : 2429-4373

Éditeur

CRECIB - Centre de recherche et d'études en civilisation britannique

Référence électronique

Mark Bailoni et Corinne Nativel, « Introduction : Entre fractures territoriales, représentations et identités culturelles: Comment appréhender le Nord britannique? », Revue Française de Civilisation Britannique [En ligne], XXV-2 | 2020, mis en ligne le 25 mai 2020, consulté le 27 mai 2020. URL : http:// journals.openedition.org/rfcb/5631 ; DOI : https://doi.org/10.4000/rfcb.5631

Ce document a été généré automatiquement le 27 mai 2020.

\section{c) (7) $९$}

Revue française de civilisation britannique est mis à disposition selon les termes de la licence Creative Commons Attribution - Pas d'Utilisation Commerciale - Pas de Modification 4.0 International. 


\section{Introduction : Entre fractures territoriales, représentations et identités culturelles: Comment appréhender le Nord britannique?}

Spatial Divides, Cultural Representations and Identities: Exploring the Meaning

of the North in Great Britain

Mark Bailoni et Corinne Nativel

1 Le clivage Nord-Sud est une image très souvent utilisée pour schématiser la géographie économique, sociale ou même culturelle, et pour modéliser les dynamismes territoriaux du Royaume-Uni. Cette représentation oppose un Nord postindustriel, soumis à des difficultés économiques et sociales, à un Sud tertiaire, riche et puissant. Il oppose un centre ancré dans la mondialisation (le Sud) à une périphérie marginalisée (le Nord). L'objectif de ce numéro de la Revue Française de Civilisation Britannique est de revenir sur cette image du clivage Nord-Sud, pour s'interroger sur sa pertinence après la crise de 2008, l'austérité et les mutations économiques récentes du pays et dans le contexte du Brexit, mais également pour analyser les éléments spatiaux, économiques, sociaux, culturels ou identitaires qui caractérisent le Nord comme un territoire particulier au sein du Royaume-Uni.

\section{Retour sur le clivage Nord-Sud, une représentation géopolitique $^{1}$}

2 L'image du clivage Nord-Sud est apparue dès le XIXe siècle dans des romans et des écrits politiques (Gaskell, Disraeli, etc.), singularisant le Nord comme un territoire spécifique, marqué par les mines et l'industrie, mais aussi célébré pour la beauté de ses paysages naturels. De ces représentations, les premières revendications régionalistes et décentralisatrices apparurent au tout début du XXe siècle, portées par différents 
cercles politiques (comme la Fabian Society) et intellectuels. Ainsi, en 1919, le géographe C.B. Fawcett, originaire du comté de Durham, estimait que «L'unité du Pays du Nord [North Country], et par conséquent le bien-fondé d'en faire une province distincte, reposent sur deux groupes de considérations, les unes économiques et les autres traditionnelles, chacune d'entre elles s'appuyant sur des faits géographiques. (...) Les traditions d'indépendance et d'initiatives du Pays du Nord se sont perpétuées jusqu'à nos jours, et soutiennent son aspiration à une autonomie gouvernementale provinciale $»^{2}$. Ses travaux, comme ceux d'E.M. Coulthard (From Tweed to Tees, 1934) ou de Cuthbert Headlam (The Three Northern Counties of England, 1939), décrivent tous le Nord à partir d'éléments économiques, historiques, culturels, folkloriques, patrimoniaux, géomorphologiques ou biogéographiques - tombant souvent dans une forme de déterminisme - qui, ensemble, permettent de définir une région « naturelle » et harmonieuse. Ils justifient également des revendications politiques, réclamant une meilleure considération politique de ce territoire.

3 Si le clivage Nord-Sud serait apparu au moment de l'industrialisation et de l'urbanisation du pays ${ }^{3}$, il aurait pris une dimension sociale dès l'entre-deux-guerres et les premières difficultés économiques des territoires industriels britanniques ${ }^{4}$. L'épisode célébré et mythifié de la Jarrow Crusade $(1936)^{5}$ demeure le symbole des problèmes sociaux très profonds que le Nord a connus à cette époque. D'ailleurs, les premières mesures gouvernementales d'assistance aux régions du Nord datent des années 1930, ouvrant la voie à une longue série de politiques publiques imaginées par les gouvernements successifs ${ }^{6}$. Les disparités sociales, en matière d'emploi, de richesse, d'éducation, de santé, d'espérance de vie, etc., se sont renforcées par la suite, avec le déclin des bassins miniers et la désindustrialisation de villes du Nord telles que Newcastle, Liverpool, Sheffield, Leeds, Manchester, Glasgow ou même Belfast, depuis la fin des années 1970.

4 La représentation du clivage Nord-Sud devient politique dans les années 1980, alors que les politiques économiques $\mathrm{du}$ gouvernement Thatcher sont particulièrement contestées dans le Nord. Une véritable fracture politique dans le pays apparait et isole un Nord majoritairement travailliste d'un Sud (en dehors du Grand Londres) conservateur. Se propage alors le sentiment que le Nord de l'Angleterre, l'Ecosse et le pays de Galles seraient victimes d'un déficit démocratique, subissant la politique d'un gouvernement conservateur que ces territoires travaillistes n'ont pas élu. Un nombre croissant de travaux universitaires ${ }^{7}$ montrent à quel point le thatchérisme a aggravé les disparités socio-économiques et le clivage Nord-Sud, mais le gouvernement nie toute réalité de cette fracture. Ces débats conduisent à un rapprochement entre élus travaillistes, universitaires et acteurs économiques locaux pour créer des courants régionalistes dans le Nord de l'Angleterre, réclamant une autre politique territoriale dans le pays, une meilleure prise en compte des spécificités régionales et surtout une décentralisation des pouvoirs.

L'arrivée au pouvoir du New Labour de Tony Blair et Gordon Brown et la mise en œuvre d'une dévolution à géométrie variable coïncide avec une période plus favorable pour l'économie britannique. Si Londres et son espace métropolitain sont les premiers bénéficiaires de cette période de croissance économique soutenue, le reste du pays en profite également, notamment certaines grandes villes du Nord comme Manchester ou Leeds. Dans ce contexte économique et politique - le sentiment de déficit démocratique n'a plus lieu d'être - la question du clivage Nord-Sud devient très secondaire dans les 
débats politiques, de même que les revendications de décentralisation. L'échec du référendum pour la création d'une assemblée régionale élue, et donc d'une vraie régionalisation politique, dans le Nord-Est en 2004 en témoigne.

Toutefois, le changement de cycle avec la crise économique de 2008 puis l'arrivée au pouvoir du gouvernement de coalition de David Cameron, qui engage le pays dans une politique d'austérité dès 2010 , réveillent les débats autour des disparités territoriales au Royaume-Uni ${ }^{8}$. Les enjeux constitutionnels et identitaires sont également relancés par les progrès électoraux du SNP en Ecosse et bien entendu par le référendum sur l'indépendance de 2014. Dans ce contexte, de nouvelles revendications décentralisatrices et régionalistes apparaissent dans le Nord de l'Angleterre ${ }^{9}$. Alors que la récession et l'austérité ont été particulièrement douloureuses pour les régions du Nord à l'économie toujours fragile ${ }^{10}$, les gouvernements Cameron ont lancé des programmes spécifiques censés renforcer l'économie du Nord et réduire les disparités territoriales (Northern Powerhouse, prolongement de la ligne à grande vitesse HS2, création d'une HS3 entre les villes du Nord, etc.) et répondre aux aspirations de décentralisation (créations des Local Enterprise Partnerships, City Deals, Combined Authorities, etc. ${ }^{11}$. Le gouvernement de Theresa May a maintenu ces différents dispositifs sans pour autant les doter des pouvoirs et des moyens financiers réclamés par les élus du Nord, laissant ainsi place à la critique et au doute. Ainsi, en visite dans le Yorkshire dans le cadre de sa campagne électorale, Boris Johnson jugeait bon de réaffirmer l'engagement gouvernemental en faveur du Nord en déclarant: "nous allons maximiser le pouvoir du Nord. Et nous allons faire en sorte que les personnes qui vivent ici soient celles qui contrôlent ce qui leur tient à cœur $»^{12}$.

Dans ce numéro, Danny McKinnon revient sur la stratégie de la Northern Powerhouse. Il montre que bien qu'ayant rallié un grand nombre d'acteurs économiques du Nord de l'Angleterre, celle-ci constitue essentiellement un outil de rhétorique gouvernementale sur l'économie spatiale et la nécessité d'un rééquilibrage. La cohérence et la concrétisation de ce projet ont été mises à mal par un manque d'engagement et de leadership des gouvernements britanniques depuis 2016.

8 Si l'importance et l'évolution du clivage Nord-Sud restent sujets à débats et à interprétations contradictoires selon les rapports et leurs commanditaires, la réalité des disparités territoriales au Royaume-Uni n'est plus contestée et tous les partis entendent trouver des solutions pour y répondre. Reste cependant à savoir à quelle échelle ces disparités sont les plus fortes et à quel niveau mettre en place des mesures pour les réduire.

\section{Qu'est-ce que le Nord?}

Le Nord apparait comme un concept spatial mouvant depuis l'origine de l'image du clivage Nord-Sud. En effet, selon les représentations, le Nord peut être défini à partir de plusieurs limites. Parfois, l'axe entre les estuaires de la Severn et de l'Humber (ou le Wash) marque la limite entre le Nord et le Sud. Certains auteurs utilisent la limite géologique s'étendant de l'estuaire de l'Exe dans le Devon à celui de la Tees dans le Nord-Est, qui sépare les uplands - caractérisés par des roches dures magmatiques et métamorphiques - au Nord-Ouest, des lowlands - caractérisés par des roches tendres sédimentaires - au Sud-Est ${ }^{13}$. Selon cette interprétation, le clivage Nord-Sud serait alors le résultat d'un déterminisme géologique et géographique. D'autres auteurs, comme 
Danny Dorling ${ }^{14}$, tracent plus précisément la limite Nord-Sud, également sur un axe Sud-Ouest/Nord-Est, à partir de données socioéconomiques. Le Watford Gap dans le Northamptonshire, où convergent des axes majeurs reliant Londres et le Nord, des voies romaines aux actuelles autoroute M1 et ligne de chemin de fer West Coast Main Line, symbolise parfois à lui seul la limite entre le Sud et le Nord (voir carte 1).

10 Parfois, le Nord ne s'étend qu'aux régions les plus septentrionales de l'Angleterre (soit un territoire regroupant le Northumberland, le comté de Durham et la Cumbria, soit l'ensemble des trois régions North-East/North-West/Yorkshire-and-the-Humber). Parfois, il comprend aussi les (West) Midlands, voire surtout les «nations périphériques » : l'Ecosse, le pays de Galles et même l'Irlande du Nord. Cette dimension très large d'un Nord britannique ne se définit alors pas à partir de critères historiques, culturels, politiques et identitaires, mais essentiellement sociaux et économiques. En effet, le Nord anglais partage avec l'Ecosse et le pays de Galles un riche passé industriel et minier, et une même expérience douloureuse de la désindustrialisation, d'une difficile reconversion et de problèmes sociaux persistants. Des ressentiments comparables vis-à-vis de Londres, de la City ou plus précisément des gouvernements conservateurs ont émergé simultanément dans ces trois territoires, les plaçant en position de défiance face à l'Etat central.

Dans sa contribution sur l'Ecosse, Edwige Camp-Pietrain met en évidence les tensions qui se cristallisent autour de la question constitutionnelle depuis les années 1970. En analysant les débats sur la dévolution ou le référendum sur l'indépendance écossaise menés au sein de la Chambre des Communes, l'article montre les dissensions grandissantes entre les députés du Nord de l'Angleterre et leurs homologues écossais. Ainsi, les réformes constitutionnelles aboutissent à un éloignement, pour ne pas dire un éclatement politique, où la proximité fondée sur des similitudes socio-économiques laisse place à des divergences et une hostilité grandissante. La perte d'une identité ouvrière commune, les rivalités fiscales et budgétaires et les préoccupations indépendantistes sont autant d'éléments qui fragilisent les alliances au sein du Nord britannique.

12 La définition spatiale du Nord pose en effet la question de son unité et de sa diversité. Peut-on considérer le Nord comme une seule et même périphérie d'un noyau métropolitain constitué par le grand Sud-Est et polarisé par Londres (voire la City) ou est-il, comme certains géographes l'affirment, un archipel dénué d'unité ${ }^{15}$ ? Derrière l'idée d'un Nord divers et multiple, apparait aussi la question de l'échelle d'analyse des disparités territoriales et des phénomènes spatiaux comme la métropolisation. En effet, s'il est clair que les indicateurs sociaux et économiques montrent des disparités claires entre le Nord et le Sud à une échelle régionale, les inégalités apparaissent parfois beaucoup plus nettes et plus profondes à une échelle locale. Il existe des poches de forte précarité au Sud, comme des espaces de prospérité au Nord ${ }^{16}$.

13 Ainsi dans son article, David Fée montre qu'au-delà des discours politiques et des préjugés, il n'est pas évident d'évoquer un réel clivage Nord-Sud à propos du logement. Les disparités en matière de nature et de qualité des logements, comme à propos de l'évolution du marché et des prix, doivent se lire à d'autres échelles, souvent selon d'autres axes de rupture qu'un simple clivage Nord-Sud.

Dans le domaine de la santé, la question du fossé Nord-Sud se pose de manière criante : les inégalités entre les régions du Nord et du Sud britanniques sont extrêmes ainsi que le révèlent de nombreux indicateurs tels que l'espérance de vie, les maladies 
chroniques ou l'état de santé psychique. Dans son article consacré à ce sujet, Louise Dalingwater souligne le lien avec l'échelon national où en dépit des principes d'égalité d'accès aux soins et de couverture universelle des services de santé, les réformes dans le champ de la protection sociale n'ont pas permis d'éradiquer la fracture Nord-Sud, bien au contraire.

Carte 1 : Définitions territoriales du Nord

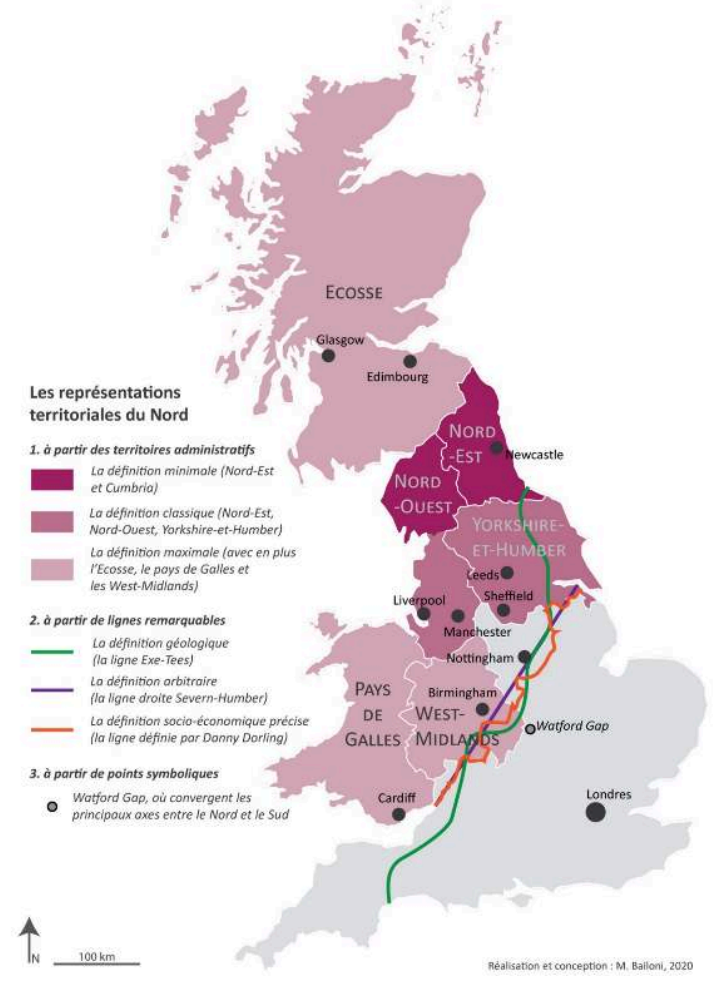

15 Au-delà d'une approche purement spatiale, le Nord se définit également par son identité et ses particularismes culturels. Le passé industriel reste à ce titre incontournable et contribue encore aujourd'hui à définir ce territoire. Si les clichés de l'industrie lourde, des mines et des cités ouvrières en déliquescence ont parfois été considérés comme des fardeaux pour le Nord, notamment dans des stratégies de reconversion économique et d'attractivité, l'histoire industrielle et minière et la mémoire ouvrière de ces territoires sont aujourd'hui célébrées et mises en valeur. La prise de conscience a été précoce au Royaume-Uni ${ }^{17}$ et le patrimoine industriel est désormais protégé et préservé, devenant même source d'une véritable attractivité touristique et moteur de renouveau urbain. Comme le montre Aurore Caignet à travers une étude comparative de deux anciens sites de l'industrie du textile à Bradford et à Manchester, ces stratégies peuvent prendre des formes très différentes. Dans ces deux villes post-industrielles, les pouvoirs publics ont choisi dans un cas de mettre en valeur le passé industriel à des fins touristiques et dans l'autre d'innover en proposant à la population locale un projet de renouveau urbain intégrant les dimensions culturelles, commerciales et résidentielles.

Le Nord se révèle également à travers ses représentations esthétiques. Les œuvres poétiques et cinématographiques soulignent à quel point le langage et l'image sont essentiels pour saisir l'essence et la pluralité de ce territoire à la fois réel et imaginé. 
Claire Hélie s'intéresse aux représentations du Nord de l'Angleterre dans la poésie contemporaine. Sous la plume de Steve Ely ou de Peter Riley, le Nord apparaît sous différentes facettes. Qu'il s'agisse de son passé industriel marqué par des violences multiformes ou des nouvelles lignes d'horizon qui se dessinent, la forme poétique construit une contre-culture esthétique qui affirme son identité propre tout en offrant la possibilité d'un rapprochement plutôt que d'un creusement du fossé Nord-Sud.

Anne-Lise Marin-Lamellet montre que depuis les années 1960, le cinéma britannique a opéré des dynamiques de mythification, de démythification et de remythification de la figure du Northerner et de son territoire. Cette figure, fortement genrée, se forge dans un contexte de désindustrialisation et de marasme économique mais aussi, et c'est le fil conducteur, dans une aversion commune pour le Sud et en particulier pour Londres qui exerce à la fois un pouvoir de fascination et de répulsion. À travers cette tension, le cinéma diffuse dans l'imaginaire collectif une identité régionale empreinte de sentiments de fierté et de dignité humaine.

\section{Quelle place pour le Nord dans le Royaume-Uni du Brexit?}

18 Si les résultats électoraux montrent classiquement une fracture Nord-Sud, les résultats du référendum sur le Brexit font ressortir des clivages beaucoup plus complexes, conséquences du caractère multifactoriel du vote leave. L'article de Matt Beech et de Kevin Hickson analyse le fossé socio-culturel révélé par le Brexit en Angleterre. Ici, le "Nord" et le "Sud" servent de métaphores pour appréhender ce clivage: sur le plan culturel, le Nord apparaît comme plus conservateur qu'un Sud plus cosmopolite. En s'appuyant sur un ensemble de données comprenant les résultats des élections partielles, locales et législatives ainsi que des statistiques issues du référendum de 2016 sur l'appartenance à l'Union européenne, les auteurs mettent en évidence le rôle fondamental joué par le Parti Travailliste de Jeremy Corbyn dont les valeurs se sont éloignées de ses traditionnels bastions électoraux du Nord.

Quelles pourraient être les conséquences du Brexit sur le Nord ? Les visions libérales et conservatrices dressent des scénarios très optimistes selon lesquels celui-ci pourrait redynamiser son industrie exportatrice par de nouveaux accords commerciaux avec, entre autres, les régions du Commonwealth. Les villes côtières pourraient quant à elles bénéficier d'un regain de contrôle sur les zones de pêche. Mais ces dynamiques pourraient bien rester illusoires sans rééquilibrage fiscal en faveur du Nord ${ }^{18}$. Bien que la géographie électorale du Brexit démente une interprétation hâtive en termes de fracture Nord-Sud, celle-ci est fortement corrélée avec sa trajectoire économique, notamment avec l'évolution du PIB par habitant sur le long cours. La fragilité et la dépendance économiques constituent des facteurs décisifs, faisant du Brexit un paradoxe pour le Nord: les régions et localités s'étant majoritairement exprimées en faveur du "leave » sont celles qui seront le plus fortement impactées par la réduction des échanges commerciaux avec les pays membres de l'Union Européenne ainsi que par le retrait des subventions que celle-ci leur accordait ${ }^{19}$. Une grande partie des fonds structurels européens (Fonds Social Européen, Fonds Européen de Développement Régional, etc.) représentant 10,6 milliards d'euros sur la période 2014-2020, revenait aux régions post-industrielles du Nord anglais, à l'Ecosse ainsi qu'au Pays de Galles et aux régions rurales du Sud-Ouest. La programmation pluriannuelle permettait de 
planifier et de mettre en œuvre de nombreux projets de développement économique et de rénovation urbaine ${ }^{20}$. De Cameron à Johnson, les gouvernements conservateurs sont restés évasifs sur la manière dont leur « nouvelle stratégie industrielle » permettrait de compenser ce gouffre financier. Le UK Shared Prosperity Fund, censé prendre le relais à compter de 2021 est essentiellement axé sur le capital humain et sur l'échelon local ce qui compromettra la capacité d'impulser d'importantes dynamiques régionales, avec une inévitable recentralisation des pouvoirs budgétaires vers Westminster.

À ces difficultés s'ajoutent les conséquences de la pandémie mondiale du Covid-19 qui au tournant des années 2020, remet un coup de projecteur sur la question des déséquilibres socio-économiques, tout particulièrement en milieu urbain. En pleine crise sanitaire, le Nord-Ouest était particulièrement touché et devançait Londres en tant qu'épicentre de la pandémie ${ }^{21}$. À l'argument d'un effet égalisateur de la crise sanitaire (leveller), on peut en effet opposer celui d'un effet "révélateur " du sousinvestissement chronique dont souffre ce territoire. À Salford et Liverpool, qui sont parmi les villes les plus impactées par les coupes budgétaires depuis 2010, les décès liés au Covid-19 sont deux à trois fois plus élevés que la moyenne nationale ${ }^{22}$. Par ailleurs, les différences structurelles sur le marché du travail accentueront très vraisemblablement le chômage, la pauvreté et la dépendance aux aides sociales dans les villes du Nord où l'on trouve davantage d'emplois précaires et une moindre proportion d'emplois stables dans le secteur des services à haute valeur ajoutée pouvant être maintenus via le télétravail ${ }^{23}$.

21 En somme, le Nord britannique constitue un objet de recherche d'une grande richesse qui ne se limite pas à l'étude du fossé Nord-Sud. Il se définit à la fois par son territoire et par les représentations qu'il véhicule. Ainsi dans la période post-Brexit et post-Covid qui s'ouvre en ce début de la décennie 2020, le Nord ne trouvera plus à la périphérie mais bien au centre des discussions et des évolutions que connaîtra le Royaume-Uni.

Ce numéro de la Revue Française de Civilisation Britannique est le prolongement du colloque interdisciplinaire "Revisiting the North-South Divide: The Changing Face of the North", organisé du 4 au 6 novembre 2016 à Nancy, par le CRECIB et les laboratoires CREW (Université Paris 3 Sorbonne Nouvelle), IDEA (Université de Lorraine) et LOTERR (Université de Lorraine). Il rassemble des articles issus de communications et nourris des échanges et des débats pendant ce colloque.

Ce numero a été mis en page et préparé pour la publication en ligne par Anne Cousson, Shirley Doulière et John Mullen.

BIBLIOGRAPHIE

BACHTLER John F. et BEGG Iain, « Cohesion Policy after Brexit: the Economic, Social and Institutional Challenges, Journal of Social Policy 46:4 (2017), pp. 1-19.

BAILONI Mark, «Le Nord de l'Angleterre : l'affirmation d'un territoire politique et identitaire », Hérodote $\mathrm{n}^{\circ} 137$ (2010), pp. 70-92. 
BAILONI Mark, « Inequalities in England: Regional Differences and New Perspectives on a Geopolitical Issue ", in Fée David et Kober-Smith Anémone (dir.), Inequalities in the UK - New Discourses, Evolutions and Actions (Bingley, Emerald, 2017), pp. 59-75.

BAILONI Mark, « Le Nord de l'Angleterre : enjeux géopolitiques d'un territoire périphérique », Outre-Terre n 49 (2017), pp. 99-111.

COULTHARD E.M., From Tweed To Tees (Edinburgh, W. \& A.K. Johnston Ltd, 1934).

COX Ed, "Devolution in England - Is the Genie out of the Lamp?" The Political Quarterly 87:4 (2016), pp. 565-571

DORLING Danny et BETHAN Thomas, People and Place - A 2001 Census Atlas of the UK (Bristol, The Policy Press, 2004).

DUNFORD Michael, "Metropolitan Polarization, the North-South Divide and Socio-Spatial Inequality in Britain”, European Urban and Regional Studies 2:2 (1995), pp. 145-170.

FAWCETT Charles Bungay, Provinces of England, A Study of Some Geographical Aspects of Devolution (Londres, Hutchinson \& Co., 1919).

FÉE David et KOBER-SMITH Anémone (dir.), Inequalities in the UK - New Discourses, Evolutions and Actions (Bingley, Emerald, 2017).

GARDINER Ben, MARTIN Ron, SUNLEY Peter et TYLER Peter "Spatially Unbalanced Growth in the British Economy”, Journal of Economic Geography 13:6 (2013), pp. 889-928.

GARSIDE Peter L. et HEBBERT Michael (dir.) British Regionalism 1900-2000 (Londres, Mansell Publishing, 1989).

GIOVANNINI Arianna, “Towards a "New English Regionalism" in the North? The Case of Yorkshire First”, The Political Quarterly 87:4 (2016), pp. 590-600.

GREEN Anne E., "The North-South Divide in Great Britain: An Examination of the Evidence", Transactions of the Institute of British Geographers 13:2 (1988), pp. 179-198.

HALL Peter, Urban and Regional Planning (Londres, Routledge, 1992).

HEADLAM Cuthbert, The Three Northern Counties of England (Gateshead, Northumberland Press, 1939).

JEWELL Helen M., The North-South Divide, the Origins of the Northern Consciousness in England, (Manchester, Manchester University Press, 1994).

KITSON Matt, MARTIN Ron, et TYLER Peter, “The Geographies of Austerity”. Cambridge Journal of Regions, Economy and Society 4:3 (2011), pp. 289-302.

LEWIS Jim et TOWNSEND Alan (dir.), The North South Divide, Regional Change in Britain in the 1980s (Londres, Paul Chapman Publishing, 1989).

LOS Bart, MCCANN Philip, SPRINGFORD John et THISSEN Mark, « The mismatch between local voting and the local economic consequences of Brexit », Regional Studies 51:5 (2017), pp. 786-799.

MAGRINI Elena « How will Coronavirus affect jobs in different parts of the country? », Centre for Cities Blog, 17 mars 2020, https://www.centreforcities.org/blog/how-will-coronavirus-affect-jobsin-different-parts-of-the-country/ [consulté le 4 mai 2020]

MCCOMBIE John S.L. et SPREAFICO Marta R.M., "Brexit and its possible implications for the UK and its regions: a post-Keynesian perspective”, Papers in Regional Science 97:1 (2018), pp. 133-149. 
MARTIN Ron, “The Political Economy of Britain's North-South Divide”, Transactions of the Institute of British Geographers 13:4 (1988), pp. 389-418.

MARTIN Ron et ROWTHORN Robert, The Geography of De-Industrialisation. (London, Macmillan, 1986).

PIDD Helen, BARR Caelainn et MOHDIN Aamma « Calls for health funding as poor bear brunt of Covid-19 ", The Guardian, 1 mai 2020, https://www.theguardian.com/world/2020/may/01/ covid-19-deaths-twice-as-high-in-poorest-areas-in-england-and-wales [consulté le 4 mai 2020]

PIKE Andy, MACKINNON Danny, COOMBES Mike, CHAMPION Tony, BRADLEY David, CUMBERS Andy, ROBSON Liz et WYMER Colin, Uneven Growth: Tackling City Decline (York, Joseph Rowntree Foundation).

PROCTOR, Kate, PARVEEN, Nazoa et PIDD, Helen « North-West overtakes London for number of Covid Hospital Cases », The Guardian, 3 mai 2020. https://www.theguardian.com/uk-news/2020/ may/03/north-west-overtakes-london-for-number-of-covid-19-hospital-cases-coronavirus [consulté le 4 mai 2020].

SCOTT Peter, Triumph of the South: A Regional History of early Twentieth Century Britain (Aldershot, Ashgate, 2007).

TAYLOR, Peter, “The Meaning of the North: England's 'foreign country' within”, Political Geography 12:2 (1993), pp. 136-155.

TOMANEY John, "Governing the Region Past, Present and Future", Conférence St Cuthbert Day, 20 mars 2003 à l'Université de Newcastle.

\section{NOTES}

1. Mark Bailoni, «Le Nord de l'Angleterre : l'affirmation d'un territoire politique et identitaire », Hérodote $\mathrm{n}^{\circ} 137$, p. 70-92, DOI : 10.3917/her.137.0070

2. "The unity of the North Country, and hence its claim to be a distinct province, rests upon two groups of considerations, the one economic and the other traditional, both of which arise from its geographic position and relations (...) The North Country traditions of independence and initiative have been carried on to our own days, and well support its claim to provincial selfgovernment ». Charles Bungay Fawcett, Provinces of England, A Study of Some Geographical Aspects of Devolution (Londres, Hutchinson \& Co., [1919] 1960) pp. 87-88.

3. Ron Martin, "The Political Economy of Britain's North-South Divide", Transactions of the Institute of British Geographers 13:4 (1988), pp. 389-418; Peter Scott, Triumph of the South: A Regional History of early Twentieth Century Britain (Alderhot, Ashgate, 2007).

4. John Tomaney, "Governing the Region Past, Present and Future", Conférence St Cuthbert Day, 20 mars 2003 à l'Université de Newcastle.

5. Du 5 au 31 octobre 1936, quelque 200 chômeurs avaient marché depuis la ville de Jarrow (Tyneside) jusqu'à Londres pour délivrer une pétition contestant le chômage et la pauvreté suite à la fermeture du chantier naval de Palmer.

6. Peter Hall, Urban and Regional Planning (Londres, Routledge, 1992).

7. Ron Martin, Op.cit.; Ron Martin et Bob Rowthorn, The Geography of De-Industrialisation (Londres, Macmillan, 1986) ; Peter L. Garside et Hebbert M. (dir.) British Regionalism 1900-2000 (Londres, Mansell Publishing, 1989) ; Anne E. Green, "The North-South Divide in Great Britain: An Examination of the Evidence", Transactions of the Institute of British Geographers 13:2 (1988), pp. 179-198 ; Jim Lewis et Alan Townsend (dir.) The North South Divide, Regional Change in Britain in the 
1980s (Londres, Paul Chapman Publishing, 1989) ; Peter Taylor "The Meaning of the North : England's 'foreign country' within”, Political Geography 12:2 (1993), pp. 136-155.

8. David Fée et Anémone Kober-Smith (dir.), Inequalities in the UK - New Discourses, Evolutions and Actions, (Bingley, Emerald, 2017).

9. Ed Cox, "Devolution in England - Is the Genie out of the Lamp?", The Political Quarterly 87:4 (2016), pp. 565-571; Adrianna Giovannini, “Towards a "New English Regionalism" in the North? The Case of Yorkshire First", The Political Quarterly 87:4 (2016), pp. 590-600.

10. Matt Kitson, Ron Martin, \& Peter Tyler, "The Geographies of Austerity", Cambridge Journal of Regions, Economy and Society 4:3 (2011), pp. 289-302; Ben Gardiner, Ron Martin, Peter Sunley et Peter Tyler, "Spatially Unbalanced Growth in the British Economy", Journal of Economic Geography, 13:6 (2013), pp. 889-928; Andy Pike, Danny MacKinnon, Mike Coombes, Tony Champion, David Bradley, Andy Cumbers, Liz Robson et Colin Wymer, Uneven Growth: Tackling City Decline (York, Joseph Rowntree Foundation, 2016).

11. Mark Bailoni, «Le Nord de l'Angleterre : enjeux géopolitiques d'un territoire périphérique », Outre-Terre $\mathrm{n}^{\circ} 49$ (2017), pp. 99-111.

12. "We are going to maximise the power of the North. And we are going to make sure that it is people here who are in control over the things that matter to them." https://www.itv.com/ news/calendar/2019-09-13/pm-boris-johnson-gets-mixed-reaction-as-he-promises-to-revitalisenorthern-powerhouse-on-visit-to-yorkshire/ [consulté le 26 avril 2020].

13. Jewell Helen M., The North-South Divide, the Origins of the Northern Consciousness in England (Manchester, Manchester University Press, 1994).

14. Danny Dorling « Persistent North-South divides » in Neil M. Coe et Andrew Jones (dir.), The Economic Geography of the UK (London, Sage, 2010), pp. 12-28.

15. Danny Dorling et Bethan Thomas, People and Place - A 2001 Census Atlas of the UK (Bristol, The Policy Press, 2004); Michael Dunford, "Metropolitan Polarization, the North-South Divide and Socio-Spatial Inequality in Britain", European Urban and Regional Studies 2:2 (1995), pp. 145-170.

16. Mark Bailoni, 2010, op. cit.; Mark Bailoni, "Inequalities in England : Regional Differences and New Perspectives on a Geopolitical Issue", in David Fée et Anémone Kober-Smith (dir.), Inequalities in the UK - New Discourses, Evolutions and Actions (Bingley, Emerald, 2017), pp. 59-75.

17. Simon Edelblutte, Paysages et territoires du patrimoine industriel au Royaume-Uni, Revue Géographique de l'Est n¹-2/48 (2009), https://journals.openedition.org/rge/1165.

18. John S.L. McCombie et Marta R.M. Spreafico, «Brexit and its possible implications for the UK and its regions: a post-Keynesian perspective », Papers in Regional Science 97:1 (2018), pp. 133-149.

19. Bart Los, Philip McCann, John Springford et Mark Thissen, «The mismatch between local voting and the local economic consequences of Brexit », Regional Studies 51:5 (2017), pp. 786-799.

20. John F. Bachtler et Iain Begg, "Cohesion Policy After Brexit: the Economic, Social and Institutional Challenges », Journal of Social Policy 46:4 (2017), pp. 1-19.

21. Kate Proctor, Nazia Parveen et Helen Pidd, « North-West overtakes London for number of Covid Hospital Cases », The Guardian, 3 mai 2020. https://www.theguardian.com/uk-news/2020/ may/03/north-west-overtakes-london-for-number-of-covid-19-hospital-cases-coronavirus [consulté le 4 mai 2020].

22. Helen Pidd, Caelainn Barr et Aamma Mohdin, «Calls for health funding as poor bear brunt of Covid-19, The Guardian, 1 mai 2020, ; https://www.theguardian.com/world/2020/may/01/ covid-19-deaths-twice-as-high-in-poorest-areas-in-england-and-wales [consulté le 4 mai 2020].

23. Elena Magrini, «How will Coronavirus affect jobs in different parts of the country? ", Centre for Cities Blog, 17 mars 2020, https://www.centreforcities.org/blog/how-will-coronavirus-affectjobs-in-different-parts-of-the-country/ [consulté le 4 mai 2020] 


\section{AUTEURS}

\section{MARK BAILONI}

Géographe, maître de conférences, Université de Lorraine / LOTERR (EA 7304)

CORINNE NATIVEL

Angliciste, maîtresse de conférences, Université Paris-Est Créteil / IMAGER (EA 3958) 